\title{
Semi-analytical solutions for cubic autocatalytic reaction-diffusion equations; the effect of a precursor chemical
}

\section{R. Alharthi ${ }^{1} \quad$ T. R. Marchant ${ }^{2} \quad$ M. I. Nelson ${ }^{3}$}

(Received 7 March 2012; revised 16 July 2012)

\begin{abstract}
Semi-analytical solutions for a cubic autocatalytic reaction, with linear decay and a precursor chemical, are considered. The model is coupled with diffusion and considered in a one-dimensional reactor. In this model the reactant is supplied by two mechanisms, diffusion via the cell boundaries and decay of an abundant precursor chemical present in the reactor. The Galerkin method is used to approximate the spatial structure of the reactant and autocatalyst concentrations in the reactor. Ordinary differential equations are then obtained as an approximation to the governing partial differential equations and analyzed to obtain semi-analytical results for the reaction-diffusion cell. Singularity theory and a local stability analysis are used to determine the regions of parameter space in which the different types
\end{abstract}

http://journal austms.org.au/ojs/index.php/ANZIAMJ/article/view/5340 gives this article, (c) Austral. Mathematical Soc. 2012. Published September 1, 2012. ISSN 1446-8735. (Print two pages per sheet of paper.) Copies of this article must not be made otherwise available on the internet; instead link directly to this URL for this article. 
of bifurcation diagrams and Hopf bifurcations occur. The effect of the precursor chemical concentration is examined in detail and some novel behaviours are identified.

\section{Contents}

1 Introduction

C512

2 The semi-analytical model

C514

3 Singularity theory and local stability

C516

4 Conclusion

C522

References

C523

\section{Introduction}

Many chemical reactions display surprising complexity due to the occurrence of oscillatory solutions and multiple steady state solutions. Oscillations occur in chemical reactions due to coupling with temperature or an autocatalyst. There are many experimental studies of chemical systems which develop oscillatory solutions (such as the Belousov-Zhabotinsky reaction) and multiple steady state solutions corresponding to breaking wave, mushroom and isola bifurcation diagrams (such as the minimal bromate oscillator and the iodate-arsenite reaction); Gray and Scott [1] provide more details of these experiments in the continuous flow, well stirred, tank reactor (CSTR). The governing equations for a CSTR comprise a system of ordinary differential equations (ODE). ODE models can be easily analyzed by the standard techniques of combustion theory. However, when diffusion is important the chemical 
reaction is governed by a system of partial differential equations (PDEs) which are unable to be analyzed by standard combustion theory.

The scheme considered, which represents cubic autocatalysis with linear catalyst decay and a precursor chemical, is

$$
\begin{aligned}
& A+2 B \rightarrow 3 B, \quad \text { rate }=\beta a b^{2}, \\
& P \rightarrow A, \quad \text { rate }=\beta k p, \\
& B \rightarrow C, \quad \text { rate }=\beta \gamma b,
\end{aligned}
$$

where the concentrations of the reactant, autocatalyst and precursor are a, $\mathrm{b}$, and $p$ respectively. The catalyst is not stable, but undergoes a simple linear decay. This allows a much wider variety of behavior in the system than does the cubic reactions alone and mimics some of the behaviours seen in the experimental studies described above.

Gray and Scott [2, 3] analysed the cubic reaction (1) in a CSTR with no precursor. They found that the model has three types of steady state bifurcation diagrams: the unique, mushroom and isola patterns. In addition, they found the parameter region where Hopf bifurcations occur and discussed their stability. Kay, Scott and Lignola [4] showed that, when an uncatalysed conversion step is added, the number of bifurcation diagrams increased to five. They found two new patterns; the breaking wave and isola breaking wave patterns. The breaking wave and the isola patterns occurred in very small regions of parameter space.

Scott [5] and Kay and Scott [6] studied (1) with no precursor, when the reactor is not well-stirred. This model is governed by a system of PDEs, hence the results were obtained numerically. Four steady state bifurcation diagrams were identified. The parameter regions, where the breaking wave and isola pattern occur, were too small to find numerically. Moreover, they found the parameter regions in which Hopf bifurcations occur. Merkin, Needham and Scott [7] considered (1) in a CSTR. The effect of the precursor chemical on the stability of limit cycles was considered in detail. 
Marchant [8] considered semi-analytical solutions for (1) with no precursor in a reaction diffusion cell. The Galerkin method was used to obtain an ODE model, as an approximation to the governing PDE system, with the tools of combustion theory used to analyze the ODE model. Singularity theory and a local stability analysis were used to find the regions of parameter space where the four main kinds of bifurcation diagram occur, together with the regions in which Hopf bifurcations occur. A good comparison was found between the results of the semi-analytical method and the numerical solutions of the governing PDE.

Section 2 presents the governing equations and uses the Galerkin method to obtain the ODEs which represent the semi-analytical model. Bifurcation patterns are also presented and described in detail. Section 3 uses singularity theory to calculate the hysteresis and isola bifurcation points. Varying the relative importance of the precursor chemical results in changes in the number of bifurcation patterns. Section 3 performs a local stability analysis of the semianalytical model. The double-zero eigenvalue and transversality degenerate Hopf points are found; hence the parameter region in which Hopf bifurcations occur is identified. Comparisons are made throughout the article between the semi-analytical results and numerical solutions of the governing PDEs.

\section{The semi-analytical model}

The cubic autocatalytic reaction with linear decay (1) is considered in a one dimensional reaction-diffusion cell. The governing PDEs are

$$
\begin{aligned}
& a_{t}=a_{x x}+\beta \mu-\beta a b^{2}, \quad b_{t}=b_{x x}+\beta a b^{2}-\beta \gamma b \\
& a_{x}=b_{x}=0 \quad \text { at } x=0, \quad a=1, \quad b=b_{0} \quad \text { at } x=1 \text { and } t=0 .
\end{aligned}
$$

The system (2) is in non-dimensional form with the concentrations of the reactant and autocatalyst given by $\mathbf{a}$ and $\mathrm{b}$, respectively. It is an open system; the reactor has a permeable boundary at $x=1$, joined to a reservoir which 
contains $\mathrm{a}$ and $\mathrm{b}$ at constant concentrations. The boundary condition at $x=0$ is a symmetry condition; an identical reservoir is located at $x=-1$. The system is characterized by four non-dimensional parameters. The ratio of the autocatalyst and reactant concentrations in the reservoir is $b_{0}$. The parameter $\beta$ is a measure of the importance of the reaction terms, compared with diffusion, while $\gamma$ is a measure of the importance of autocatalyst decay, compared with the cubic reaction. $\mu$ is a non-dimensional measure of the supply of the reactant $a$, from the decay of the precursor chemical. We assume that the supply of precursor in the reactor is much greater than the concentrations of $a$ and $b$; this is the pooled chemical approximation [7].

The Galerkin method requires that assumptions be made about the spatial structure of the concentration profiles, which then allows the governing PDEs (2) to be approximated by ODEs. The method requires that the exact concentrations be approximated by a series of orthogonal basis functions. The expansion

$$
\begin{aligned}
& a(x, t)=1+a_{1}(t) \cos (\pi x / 2)+a_{2}(t) \cos (3 \pi x / 2), \\
& b(x, t)=b_{0}+b_{1}(t) \cos (\pi x / 2)+b_{2}(t) \cos (3 \pi x / 2),
\end{aligned}
$$

represents the two term method used here. Expansion (3) satisfies the boundary conditions in (2), but not the governing PDE. We assume this approximation is sufficient and the bifurcation structure of the approximation is similarly close to that of the exact solution. The free parameters in (3) are found by evaluating averaged versions of the governing equations, weighted by the basis functions. This procedure gives the ODEs

$$
\begin{aligned}
\frac{d a_{1}}{d t} & =-N_{1} \beta-\frac{1}{4} a_{1} \pi^{2}, \\
\frac{d a_{2}}{d t} & =-N_{2} \beta-\frac{9}{4} a_{2} \pi^{2}, \\
\frac{d b_{1}}{d t} & =N_{1} \beta-\frac{1}{4} b_{1} \pi^{2}-\beta \gamma\left(\frac{4 b_{0}}{\pi}+b_{1}\right)+\beta \frac{4 \mu}{\pi}, \\
\frac{d b_{2}}{d t} & =N_{2} \beta-\frac{9}{4} b_{2} \pi^{2}-\beta \gamma\left(b_{2}-\frac{4 b_{0}}{3 \pi}\right)-\beta \frac{4 \mu}{3 \pi},
\end{aligned}
$$


where

$$
\begin{aligned}
& N_{1}=\frac{16}{15} \frac{a_{1} b_{0} b_{2}}{\pi}+\frac{16}{15} \frac{a_{2} b_{0} b_{1}}{\pi}+\frac{16}{3} \frac{a_{1} b_{0} b_{1}}{\pi}+\frac{144}{35} \frac{a_{2} b_{0} b_{2}}{\pi}+\frac{16}{15} \frac{b_{1} b_{2}}{\pi} \\
& +a_{2} b_{1} b_{2}+\frac{1}{2} a_{1} b_{1} b_{2}+4 \frac{b_{0}^{2}}{\pi}-4 \frac{\mu}{\pi}+a_{1} b_{0}^{2}+\frac{3}{4} a_{1} b_{1}^{2}+\frac{8}{3} \frac{b_{1}^{2}}{\pi} \\
& +\frac{72}{35} \frac{b_{2}^{2}}{\pi}+\frac{1}{2} a_{1} b_{2}^{2}+2 b_{0} b_{1}+\frac{1}{4} a_{2} b_{1}^{2} \\
& N_{2}=\frac{144}{35} \frac{a_{1} b_{0} b_{2}}{\pi}+\frac{144}{35} \frac{a_{2} b_{0} b_{1}}{\pi}+\frac{16}{15} \frac{a_{1} b_{0} b_{1}}{\pi}-\frac{16}{9} \frac{a_{2} b_{0} b_{2}}{\pi}+\frac{144}{35} \frac{b_{1} b_{2}}{\pi} \\
& +a_{1} b_{1} b_{2}-\frac{4}{3} \frac{b_{0}{ }^{2}}{\pi}+\frac{4}{3} \frac{\mu}{\pi}+\frac{1}{4} a_{1} b_{1}{ }^{2}+\frac{8}{15} \frac{b_{1}{ }^{2}}{\pi}-\frac{8}{9} \frac{b_{2}{ }^{2}}{\pi}+\frac{1}{2} a_{2} b_{1}{ }^{2} \\
& +a_{2} b_{0}{ }^{2}+\frac{3}{4} a_{2} b_{2}^{2}+2 b_{0} b_{2} \text {. }
\end{aligned}
$$

The series (3) was truncated after two terms. It is found that a two term method gives sufficient accuracy without excessive expression swell.

Figure 1 shows steady state bifurcation diagrams. Shown is the steady state of the autocatalyst concentration at the reactor's centre $(x=0), b$, versus the bifurcation parameter $\beta$. Four of the five bifurcation patterns are shown. They are (a) the unique pattern, (b) the breaking-wave pattern, (c) the mushroom pattern, and (d) the isola pattern. The one term and two term semi-analytical solutions plus the numerical solution of (2) are shown. The two term solution is very close to the numerical solution, hence it is an accurate approximation to the full system.

\section{$3 \quad$ Singularity theory and local stability}

Singularity theory allows a complete description of all qualitatively distinct behaviour, which occurs in a particular system of ODEs. The application of singularity theory to chemical reactions was described by Balakotaiah and Luss [9]. They gave conditions for the hysteresis and isola bifurcation 


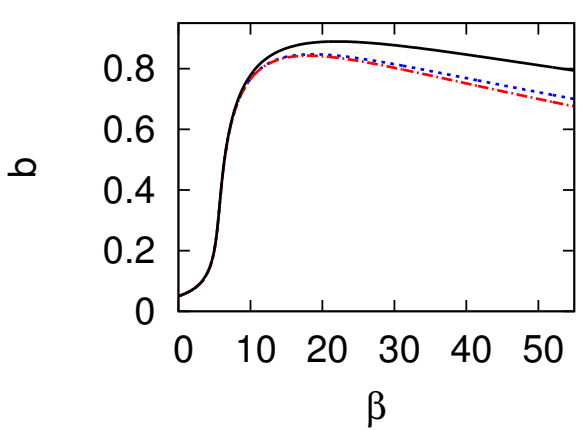

(a)

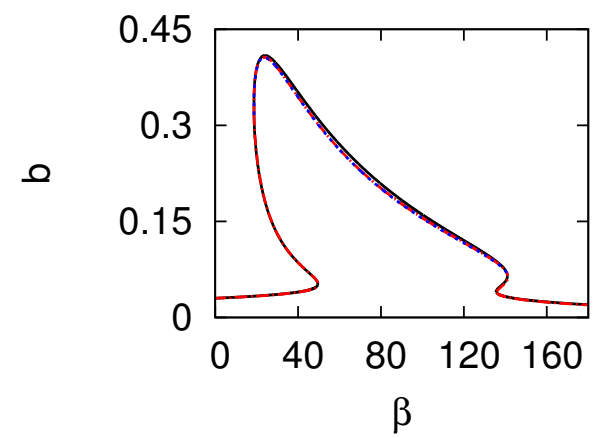

(c)

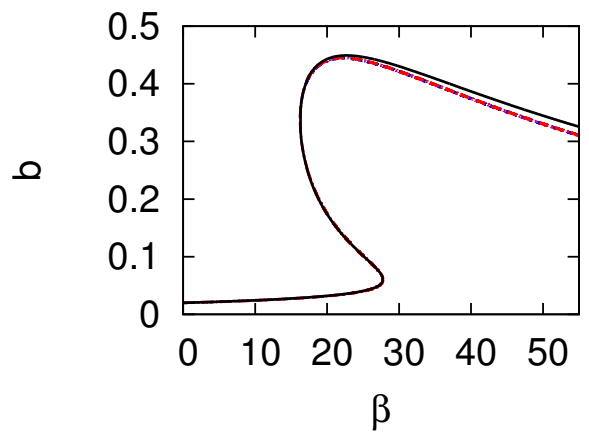

(b)

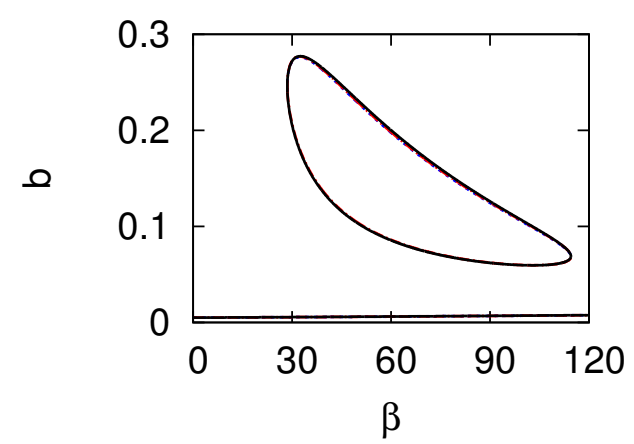

(d)

Figure 1: Steady state bifurcation diagrams of the autocatalyst concentration, $\mathrm{b}$ versus $\beta$ : (a) the unique pattern, with $\mathrm{b}_{0}=0.05, \gamma=0.018$; (b) the breaking-wave pattern with $b_{0}=0.02, \gamma=0.065$; (c) the mushroom pattern, with $b_{0}=0.03, \gamma=0.069$; (d) the isola pattern, with $b_{0}=0.005, \gamma=0.078$. The one term (black solid lines) and two term (red large dashes) semi-analytical solutions plus the numerical solution of (2) (blue small dashes) are shown. 
curves and apply the theory to examples involving first order, non-isothermal reactions in a CSTR. Singularity theory is applied to the semi-analytical model of section 2, which provides a semi-analytical description of the parameter regions in which each of the bifurcation pattern occurs. The equations corresponding to the steady state, two term model, have the general form

$$
f_{i}\left(b_{0}, b_{1}, b_{2}, a_{1}, a_{2}, \beta, \gamma, \mu\right)=0, \quad i=1,4,
$$

where $\beta$ is our choice of bifurcation parameter. The hysteresis bifurcation corresponds to solutions for which a hysteresis loop or fold first occurs in the bifurcation diagram. This corresponds to the conditions

$$
\frac{d \beta}{d b_{1}}=\frac{d^{2} \beta}{d b_{1}^{2}}=0 .
$$

The application of the condition (8) to (7) was described by Marchant [8] and leads to a set of transcendental equations, which represent lines in the $\gamma-b_{0}$ plane. The loci of hysteresis points, when crossed, causes a hysteresis loop to be created or destroyed in the relevant bifurcation diagram. The sets of transcendental equations for the hysteresis bifurcation points are found and solved using Maple. A similar process is followed in the remainder of the article to convert the mathematical conditions for the isola bifurcation curve, the isola cusp point and the double-zero eigenvalue (DZE) and transversally Hopf degeneracies into sets of transcendental equations, which are also solved numerically. The isola bifurcation points are defined by

$$
\frac{d \beta}{d b_{1}}=\frac{d \gamma}{d \beta}=0 .
$$

Again, these conditions represent lines in the $\gamma-b_{0}$ plane which, when crossed, cause an isola to be created or destroyed in the relevant bifurcation diagram; Marchant [8] gave further details.

Now consider the effect of varying the precursor chemical, via the parameter $\mu$, on the location of the bifurcation patterns. Figure 2 shows the division of the 
(a)

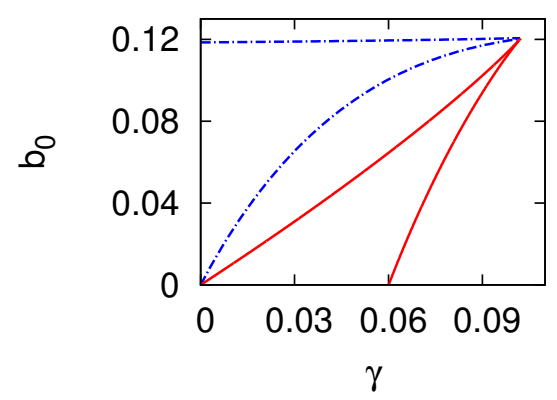

(c)

(e)
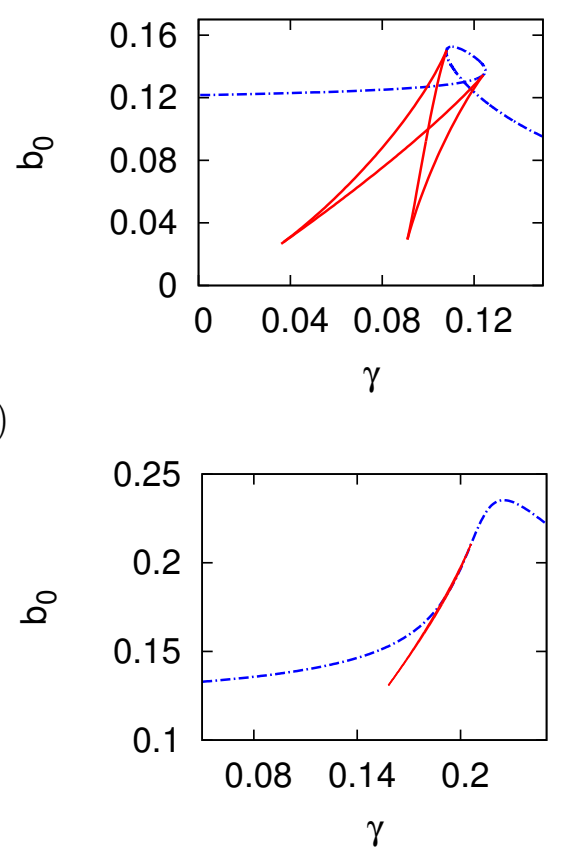

(b)

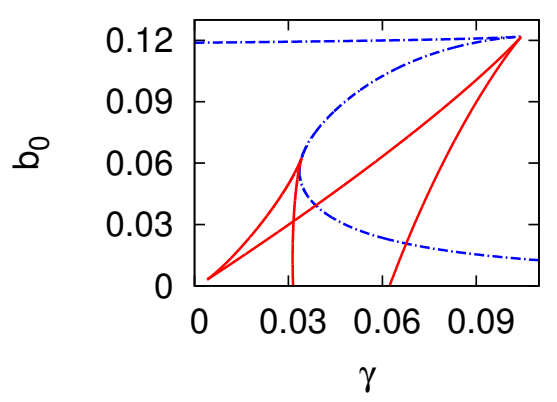

(d)

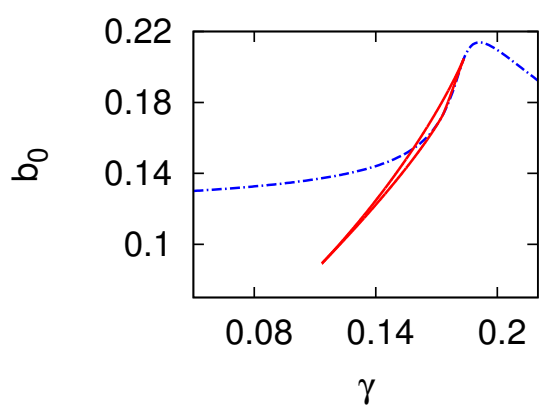

(f)

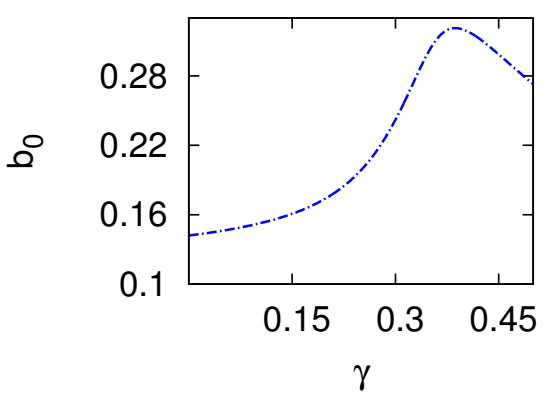

Figure 2: Division of the $\gamma-b_{0}$ plane into regions corresponding to the different bifurcation diagrams: (a) $\mu=0$, (b) $\mu=0.001$, (c) $\mu=0.01$, (d) $\mu=0.03$, (e) $\mu=0.04$ and (f) $\mu=0.1$. The isola curves (red solid lines) and the cusp curves (blue small dashes) are shown. 
$\gamma-b_{0}$ plane into regions corresponding to the different bifurcation diagrams. When $\mu=0$ there are four regions in the plane, $\mu=0.001$ has eight regions, $\mu=0.01$ has ten regions, $\mu=0.03$ has five regions, $\mu=0.04$ has five regions, and $\mu=0.1$ has two regions. When $\mu=0$ there are four kinds of steady state bifurcation diagrams, which occur in the four different regions of Figure 2(a): they are the unique, breaking-wave, mushroom and the isola pattern. For large $\mu$ only the unique and the breaking wave pattern occur, see Figure 2(f). For intermediate values of $\mu$ the division of the parameter space is very complicated; many additional regions occur and particular bifurcation patterns can occur in more than one region. The simplicity of the parameter space division for large $\mu$ is due to the disappearance of the isola curve when $\mu \approx 0.05$. The cusp curve remains which divides the plane into two regions.

Hopf bifurcations and stable oscillatory solutions occur for the system (1), both in the CSTR and the reaction-diffusion cell. The theory of Hopf bifurcations can be found in standard texts on bifurcation theory and dynamical systems such as those by Guckenheimer and Holmes [10] or Golubitsky and Schaeffer [11]. To determine the parameter region in which Hopf bifurcations occur, the points for which the bifurcation is degenerate must be found. The degenerate Hopf points are defined by

$$
\text { DZE }: f_{i}=\operatorname{tr} J=\operatorname{det} J=0, \quad H 2: f=\operatorname{tr} J=\frac{d \operatorname{tr} J}{d \beta}=\frac{d f_{i}}{d \beta}=0, \quad i=1,2 .
$$

For the two term semi-analytical model, there are a set of four ordinary differential equations (5). The eigenvalues of the Jacobian matrix are described by the quartic equation, $\lambda^{4}+\alpha_{1} \lambda^{3}+\alpha_{2} \lambda^{2}+\alpha_{3} \lambda+\alpha_{4}=0$. Hopf bifurcations occur for this system when one pair of eigenvalues is purely imaginary, which implies $q=\alpha_{4} \alpha_{1}^{2}+\alpha_{3}^{2}-\alpha_{1} \alpha_{2} \alpha_{3}=0$. The degenerate Hopf points are

$$
\text { DZE }: f_{i}=\alpha_{3}=\alpha_{4}=0, \quad H 2: f_{i}=q=\frac{d q}{d \beta}=\frac{d f_{i}}{d \beta}=0, \quad i=1,4 .
$$

By solving the sets of transcendental equations associated with the DZE and $\mathrm{H} 2$ conditions, curves in the $\gamma-\mathrm{b}_{0}$ plane are obtained. 


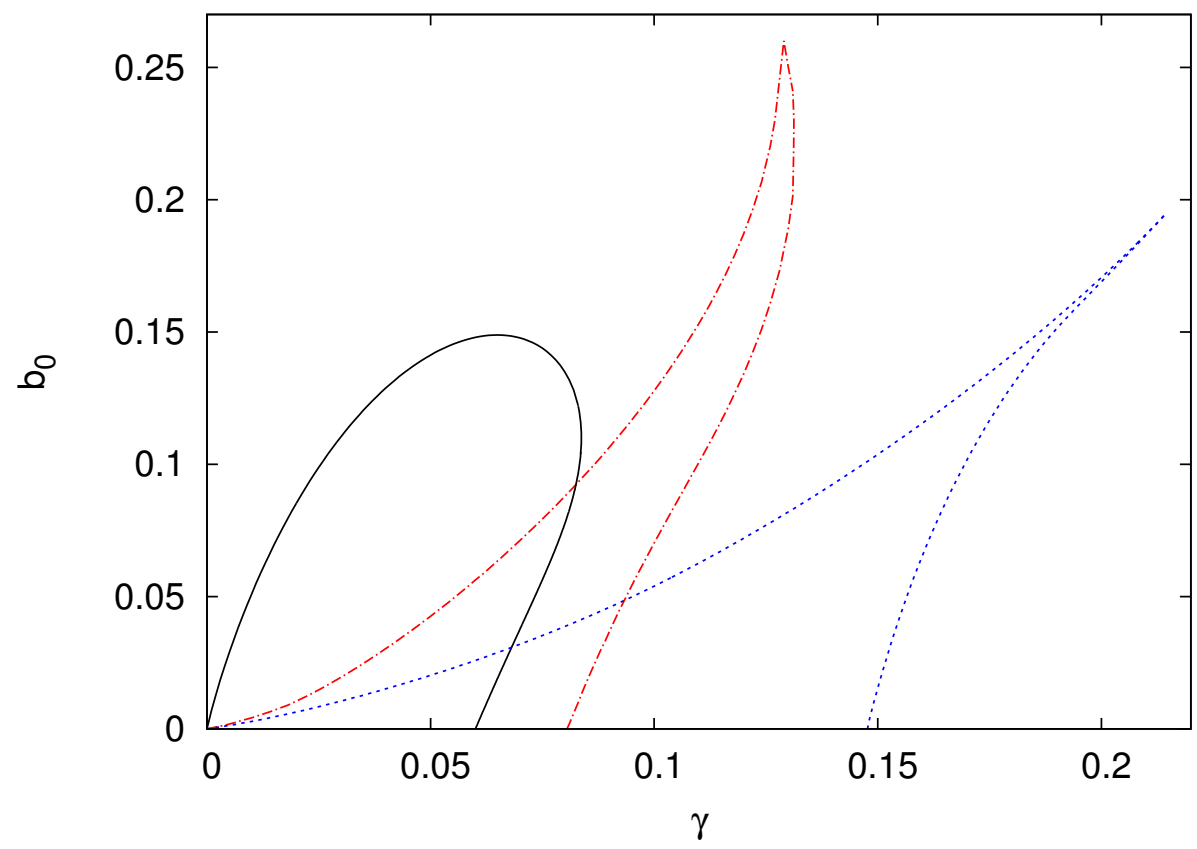

Figure 3: Hopf curve for $\mu=0$ (black solid line), $\mu=0.01$ (red dot-dashes) and $\mu=0.05$ (blue dashes)

Figure 3 shows the degenerate Hopf points, in the $\gamma-b_{0}$ plane, for $\mu=0$, 0.01 and 0.05 . For non-zero $\mu$ the region of parameter space moves in a complicated manner; for non-zero $\mu$ Hopf bifurcations are possible at large values of $b_{0}$ and $\gamma$, than for the case with no precursor. $\mu$ appears to be a useful control parameter, to stabilize or destabilize, the autocatalytic reaction.

Figure 4 shows the limit cycle curve, $a$ versus $b$ when $\mu=0.001, \beta=192.3$, $\mathrm{b}_{0}=0.08$ and $\gamma=0.05$. The one term, two term and the numerical solution of the PDEs are shown. The two term semi-analytical approximation is fairly close to the numerical solution of the PDE. 


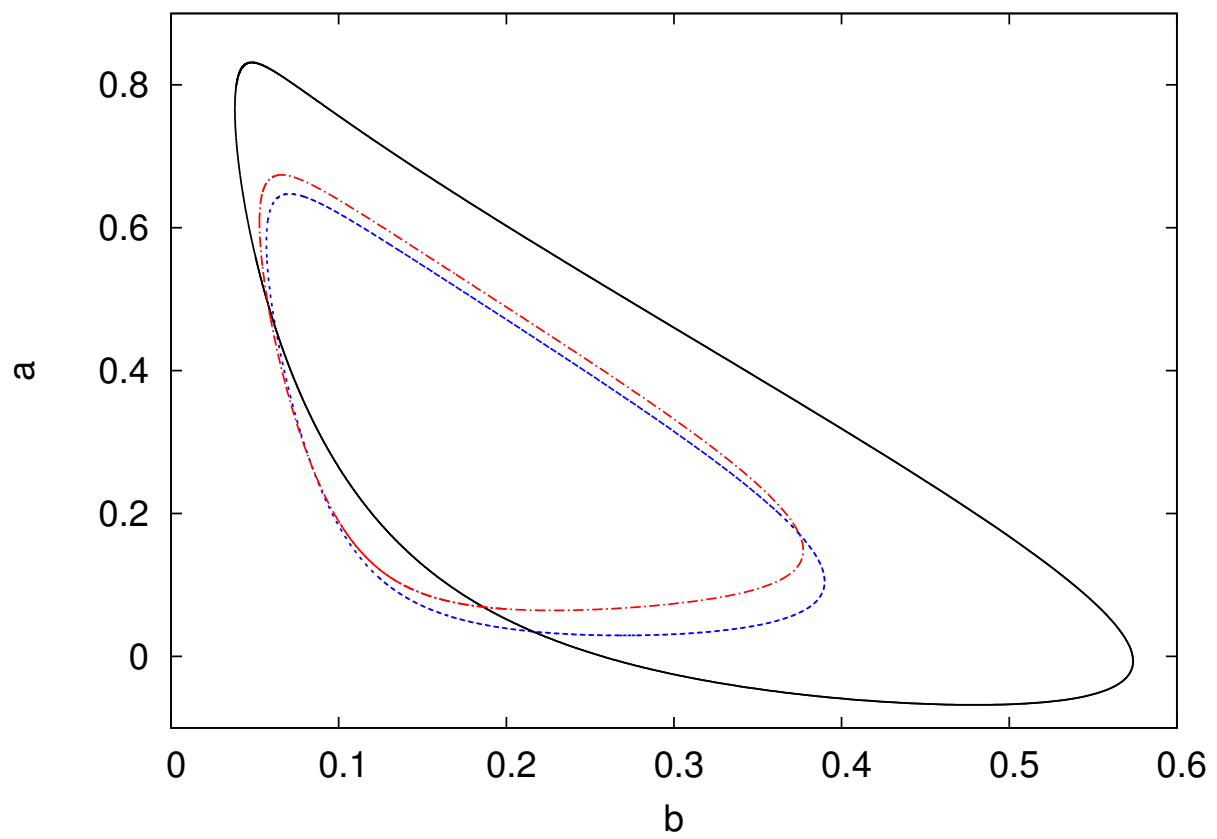

Figure 4: The limit cycle when $\mu=0.001, \beta=192.3, b_{0}=0.08$ and $\gamma=0.05$, The one term (black solid line) and two term (red large dashes) semi-analytical solutions and the numerical solution of (2) (blue small dashes) are shown.

\section{Conclusion}

Semi-analytical solutions were developed to show the effect of a precursor chemical on the cubic autocatalytic reaction-diffusion cell. The effect of varying $\mu$, on the distribution of bifurcation patterns, and the occurrence of oscillatory solutions is dramatic. The semi-analytical method proves to be a useful, accurate and robust technique for analyzing the dynamics of reaction-diffusion cells. 
Acknowledgement Alharthi gratefully thanks Taif university in Saudi Arabia for awarding him a PhD scholarship to study at the University of Wollongong, Australia.

\section{References}

[1] P. Gray and S. K. Scott. Chemical oscillations and instabilities: nonlinear chemical kinetics. Oxford University Press, London, 1990. C512

[2] P. Gray and S. K. Scott. Autocatalytic reactions in the isothermal continuous, stirred tank reactor: isolas and other forms of multistability. Chem. Engng. Sci., 38:29-43, 1983. doi:10.1016/0009-2509(83)80132-8 C513

[3] P. Gray and S. K. Scott. Autocatalytic reactions in the isothermal continuous, stirred tank reactor: oscillations and instabilities in the system $a+2 b \rightarrow 3 b ; b \rightarrow c$. Chem. Engng. Sci., 39:1087-1097, 1984. doi:10.1016/0009-2509(84)87017-7 C513

[4] S. R. Kay, S. K. Scott, and P. G. Lignola. The application of singularity theory to isothermal autocatalytic reactions: the influence of uncatalysed reactions. Proc. Roy. Soc. Lond A, 409:433-448, 1987. doi:10.1098/rspa.1986.0015 C513

[5] S. K. Scott. Isolas, mushrooms and oscillations in isothermal, autocatalytic reaction-diffusion equations. Chem. Engng. Sci., 42:307-315, 1987. doi:10.1016/0009-2509(87)85060-1 C513

[6] S. R. Kay and S. K. Scott. Multiple stationary states, sustained oscillations and transient behavior in autocatalytic reaction-diffusion equations. Proc. Roy. Soc. Lond A, 418:345-364, 1988. doi:10.1098/rspa.1988.0088 C513 
[7] J. H. Merkin, D. J. Needham, and S. K. Scott. On the creation, growth and extinction of oscillatory solutions for a simple pooled chemical reaction scheme. SIAM Journal on Applied Mathematics, 47:1040-1060, 1987. http://www.jstor.org/stable/2101706 C513, C515

[8] T. R. Marchant. Cubic autocatalytic reaction-diffusion equations: semi-analytical solutions. Proc. Roy. Soc. Lond A, 458:873-888, 2002. doi:10.1098/rspa.2001.0899 C514, C518

[9] V. Balakotaiah and D. Luss. Multiplicity features of reacting systems. Chem. Engng. Sci., 38:1709-1721, 1983.

doi:10.1016/0009-2509(83)85028-3 C516

[10] J. Guckenheimer and P. Holmes. Nonlinear oscillations, dynamical systems, and bifurcations of vector fields. Springer-Verlag, New York, 1983. C520

[11] M. Golubitsky and D. G. Schaeffer. Singularites and groups in bifurcation theory. Springer-Verlag, New York, 1985. C520

\section{Author addresses}

1. M. R. Alharthi, School of Mathematics, University of Taif, SAUdi ARABIA

mailto:mraah7610uowmail.edu.au

2. T. R. Marchant, School of Mathematics and Applied Statistics, University of Wollongong, N.S.W. 2500, AUstRALIA mailto:tim_marchant@uow.edu.au

3. M. I. Nelson, School of Mathematics and Applied Statistics, University of Wollongong, N.S.W. 2500, Australia mailto:mark_nelson@uow. edu.au 